\title{
SENSOR DATA OPTIMIZED BY FUZZY- NEURON WITH BAT COMPUTATION BASED MACHINE LEARNING ROUTING ENABLED IN WIRELESS NETWORK
}

\author{
C. Sudha ${ }^{1}$, D.Suresh ${ }^{2}$, A. Nagesh ${ }^{3}$ \\ ${ }^{1}$ Research Scholar, Annamalai University, Chidambaram, Tamilnadu, India. \\ ${ }^{2}$ Assistant Professor, Dept. of IT, Annamalai University, Chidambaram, Tamilnadu, India \\ ${ }^{3}$ Professor, Dept. of CSE, Mahatma Gandhi Institute of Technology, Hyderabad, India. \\ ${ }^{1}$ csudhahyd@gmail.com, ${ }^{2}$ deiveekasuresh@gmail.com, ${ }^{3}$ akknagesh@rediffmail.com
}

\begin{abstract}
Depending on the changes in the network and the nature of the data perceived in the network, the machine learning system has been developed to retain energy and prolong sensor life. Also, the sensor network is created by combining machine learning with the Enhanced BAT(EBAT) computational method to reduce network redundancy by aggregating the same kind of data perceived in the sensor network within a coverage range. Also, the neighbor selection is made so that the sensors' energy is used sparingly throughout the network, for which feature sets are intercepted in fuzzy-neuron machine learning mode. Also, in the network, the energy consumption of the sensor is calculated depending on the speed of the packets being sensed and the sensing interval between them. Furthermore, in the aggregation method, which is calculated to reduce energy use, the data is opened and aggregated using the neural network method. When this is done, the noise in the data is removed, and the same data is aggregated. This saves energy and increases the use of network resources. Also, the routing path is constructed using Enhanced BAT computation to build a consistent and energy-efficient path.
\end{abstract}

Keywords: sensor network; Redundancy; Aggregated; Fuzzy-Neuron; EBAT Routing

\section{Introduction}

The energy consumption of the sensor network during their interactions should be closely monitored. That is why we use some methods in the operation of this network. One of them is selecting sensors adaptable to the network area conditions, monitoring changes in the environment where the sensors are located, processing information according to those changes, and sending it to the destination. The actions of the sensors vary depending on the coverage distance of the location and the temporarily changing circumstances. To deal with these conditions, each sensor selects a set of neighbors within its coverage area. Having a small number of sensors in that neighborhood set can aid in dealing with network changes. Similarly, the selected sensors protect the network resources when sending information regularly according to changing circumstances with the correct data rate.

When converting sensors into data groups, the group varies according to the environment of the sensing area. In particular, the data should be grouped according to the data and its features. Otherwise, different sensors may combine to form an infeasible cluster set. The fuzzy inference system optimizes this problem. The time the data sense is made, its location, the distance between the two sensors, etc., are calculated. In addition, we calculate the energy costs between them, pass them on to the neural network, defuse the accurate data reports obtained from it, and group the data according to the features and variations of the data. Optimized solutions are found by selecting the inputs of the hidden neural layer and assigning weights to tune their parameters. To further optimize this, the machine learning method improves the speed of finding solutions. The BAT computation method is used additionally to optimize this learning and select routing for data transmission. This computation method is the method of an intelligent bird with global search capability. It focuses on improving machine input learning and its search. In this path selection, the sensor uses the remaining energy, its distance to transmit data, and other factors. The sensors that work on it increase the maximum coverage area, save network resources, and prolong the sensor's life by consuming less energy. We see that the performance of the network increases as the path selection takes place. 
Section 2 of this paper gives details of previous research papers on changes in sensor network routing. Also, in section 3, the proposed method, FN-EBAT, sensor data optimized by fuzzy neuron with BAT computation-based machine learning routing enabled in a wireless network, is discussed in detail. Finally, section 4 describes the results and discussions of the FN-EBAT. In the end, the conclusion of this paper is stated and its future work is expressed.

\section{Literature Survey}

Electrical load monitoring data with wireless sensor networks play a vital role in electrical power operation. Accurate load forecasts will lead to proper operation and planning for the power system, thus achieving a lower operating cost and higher reliability of electricity supply [4]. WSNS can be used to monitor the exciting region using multi-hop communication. Coverage is a primary metric to evaluate the monitoring capacity. Connectivity can also be guaranteed so that the BS can receive all the sensed data for future processing [1]. We believe that the underlying architecture must provide a low overhead execution environment for fundamental macro program primitives while naturally supporting rich extensions based on the system's operational requirements or application domain [8]. The anticipated applications for WSNs range broadly from homeland security and surveillance to habitat and environmental monitoring. Indeed, advances in microelectronics and wireless communications have made WSNs the predicted panacea for attacking a host of large-scale decision and information-processing tasks [12]. A sensor node may vary in dimensions, and the cost of sensor nodes is also variable. The physical arrangement of WSN may be like a star network or perhaps a multi-hop wireless mesh network [17]. Due to the error-prone sensor readings and abnormal environmental conditions affecting the virtual sensor predictions, the former was necessary. The primary function of the proposed system is to ensure the robustness of WSNs by providing that damaged or compromised nodes in these systems can be replaced by these machine learning-based virtual sensors [13]. Transmitting all data back to a base station for processing and making inferences is simply impossible due to the sensor's limited energy and bandwidth constraints.

As a result, Machine Learning algorithms must be applied to the WSN [2]. Clustering is the process that divides the network into interconnecting substructures called clusters. Every cluster has a cluster head that acts as a coordinator within the substructure. The cluster head acts as a medium for transferring data between the nodes. $\mathrm{CH}$ communicates with each other by using gateway nodes [7]. We designed a real-time monitor which can perform deep learning-based analysis within the deadline, considering a time-constrained environment. Simulation using the published data and the deep learning performance under time constraints are reported and discussed to assure the effectiveness of the proposed technique [15].

The Neural Network is an arithmetic algorithm to learn the complicated mapping between input and output. Data aggregation is collecting and processing sensor data to reduce network data transmission [3]. A WSN detects and measures several physical or environmental conditions such as multimedia, infrared energy emitted from objects and converted to temperature, pollutant levels, ultrasound in medical imaging, vibrations, security and surveillance, agriculture, and many other such conditions [14]. Sensor nodes waste a lot of their energy on data communication. So, reducing unnecessary transmission will help minimize energy waste and extend the network's lifetime [5]. However, the lifetime of sensor networks is limited. Therefore, there is a need to re-energize the sensor network by adding more nodes. These nodes will be equipped with more energy than the already in-use nodes, creating heterogeneity in terms of node energy [9]. This TDMA scheduling saves energy and extends the network lifetime. Further, the $\mathrm{CH}$ fuses the data to filter out any redundant information and then transmits it to the BS. Sensor networks placed in distant geographical locations like animal tracking should tolerate critical conditions to survive long-term deployments [11]. Swarm intelligence algorithms denote a group of meta-heuristic algorithms that use specific evolutionary steps to simulate swarm intelligence found in nature. The gathering of data is quite common, but it may have some critical operations to perform in various applications of the WSN [6]. For example, accumulating cloud gaming in the multiplayer cooperative scenario to overcome data synchronization concerning its cloud data [16]. Machine learning algorithms can rapidly and efficiently establish accurate localization of nodes to overcome the dynamic nature of network organization due to external or internal factors. In contrast, the improved Pliable Cognitive Medium Access protocol proposed in this study maintains the conventional characteristics of the end-sensor node, copes with the required QoS for emerging wireless sensor applications, and allows more efficient radio spectrum utilization [10].

\section{Proposed Work}

\subsection{FN-EBAT Design and Implementation}

The network contains a set of sensors $S=\left(\begin{array}{llll}s_{1} \ldots s_{2} & \ldots s_{n}\end{array}\right)$ within its topography boundaries. Also, each sensor holds a collection of neighbors within its coverage range as $N=\left(n_{1} \ldots n_{2} \ldots n_{n}\right)$. The sensor randomly senses and stores the data in a table format called node list $N_{L}$. In $N_{L}$, the sensor saves information about the data along with essential features and neighbor details. Because each feature of the data is stored $N_{L}$, it is possible to 
know the exact details. In that list, along with the feature set $F_{S}$, each sensor stores the node's ID that sensed the data $S_{D}$, the sensor's location L, the time $t$ it sensed the data, and the counts $S_{C}$ of the packets that the sensor sensed. With details of these $F_{S}$, it is possible to know the conditions of the perceived area and the variations that frequently occur in that region.

Perhaps, when monitoring more data variations from the feature set $F_{S}$, it is necessary to check the correct variations in each data to know the type of application perceived. This allows the network to understand the situation of the area where the sensor is located. Furthermore, the nature of the data can be understood by grouping and storing the identical data collected in one place based on its $F_{S}$. Thus, a fuzzy inference $F_{I}$ method is used to group data $D_{G}$ with consensus features.

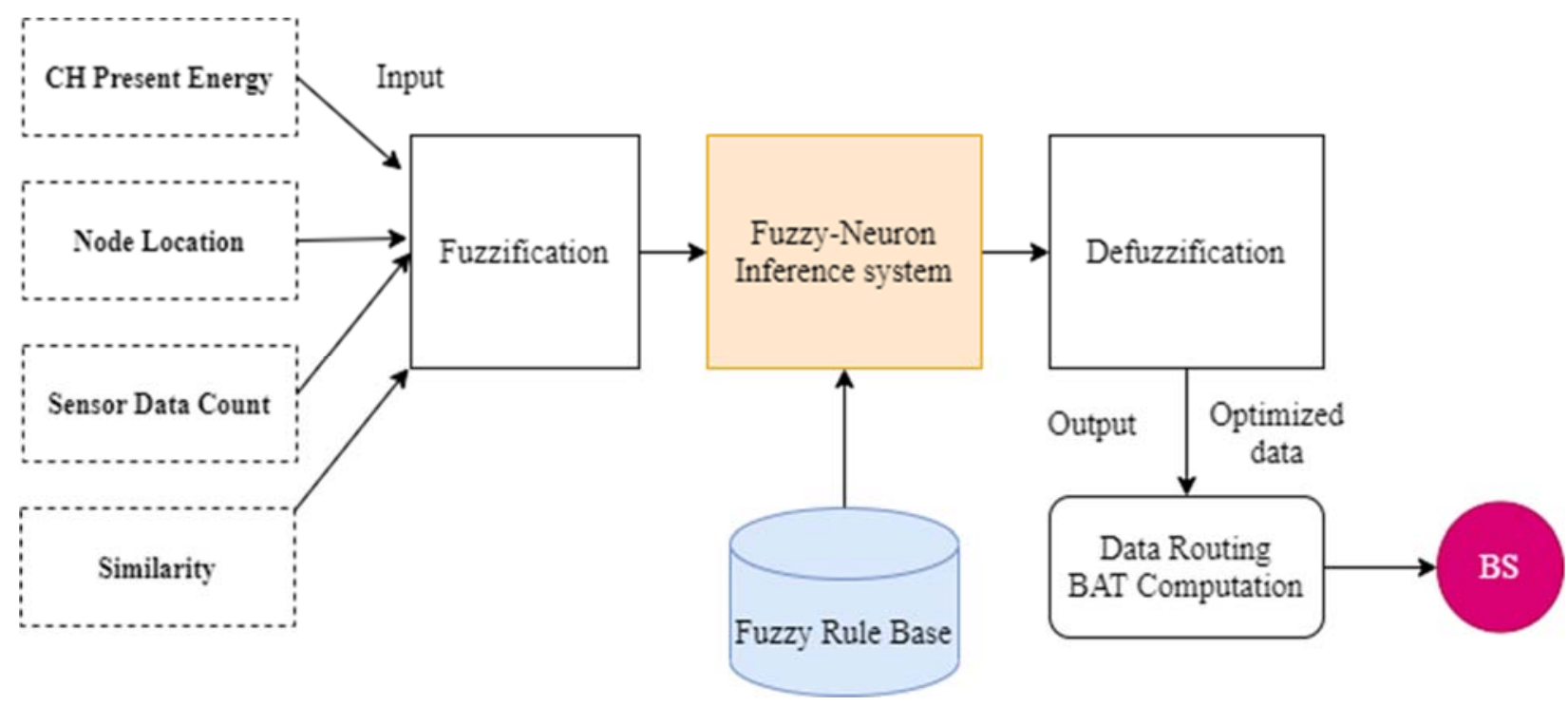

Fig.1. Fuzzy-Neuron and EBAT Computation(FN-EBAT) Architecture

Figure 1 depicts the design of the Fuzzy-Neuron based optimization system for the suggested model known as FN-EBAT. The input layer provides four inputs, the hidden layers provide 256 rules, and the output layer provides one output. We used four linguistic variables in our system, each with three levels and the proposed parameter Similarity.

In calculating this $F_{I}$, we must first give the features of the $S_{D}$ as input in the fuzzification. They are data sensed time $D_{S t}$, node location $D_{S L}$, sensed data $S_{D C}$ count, etc. To predict changes in these $F_{S}$, data differences between current data and previous data are calculated below; along with the data classification, we validated the sensed data time difference $S_{D T}$ and location difference $S_{D L}$ as given below.

$$
\begin{aligned}
& F_{S}=\left\|S_{D S}-S_{D-1}\right\| \\
& S_{D T}=\left\|D_{S t}-D_{S t-1}\right\| \\
& S_{D L}=\left\|D_{S L}-D_{S L-1}\right\|
\end{aligned}
$$

In a node, once sensed data, the existing $F_{S}$ indicate the sensor's minute time status. These parameter variations will be constantly monitored and updated in the $F_{I}$ set. Depending on the sensing application, many DG are formed, and the data will be updated within the respective group. This $D_{G}$ contains various sensing data, and this is because, in today's environment, sensors can sense a variety of data. If the data groups formed in it are further classified using cost $C$ estimation as given below to distinguish the accurate data in it as shown below. Here $S_{D}$ is the sensed data from the $D_{G}$, and $M_{D}$ is the random middle data of the stored $N_{L} F_{S}$, from this we are finding the minimum and maximum $C$ value.

$$
C=\sum D_{G}\left(S_{D}, M_{D}\right) \sum F_{S}\left(S_{D}, M_{D}\right)
$$


The stored data must be processed in the fuzzy rule in all the existing groups, thereby separating their ranges and specifying a membership size $M_{S}$.

$$
M_{S}=\frac{\sum D_{G} F_{S} S_{D}}{\sum D_{G} F_{S}}
$$

We need to keep this membership size and the separated features to form a more accurate data group when doing formation. Then, according to the Lagrangian multiplier calculation, more accurate data classification $D_{C}$ can be done to separate the maximum and lowest values for the $S_{D}$ set and form an $D_{G}$. $M_{S}$ Provides each $S_{D}$ classification value, according to the value $D_{G}$ will renew at each round, also the middle data point $M_{D i}$ computed as below:

$$
M_{D i}=\frac{1 / D_{G}}{\sum D_{G}\left(\frac{1}{D_{G}}\right)}
$$

According to the above computations, each sensed data is streamlined at each sending time. With the accurate data thus obtained, the clustering of the individual $D_{G}$ is formed according to the values of their respective features; this process will continue till the end of network execution. Once the sensors have sensed the data according to their location and location, they can collect the data, divide their features, form the $D_{G}$ accordingly, and know the exact time and situation of that particular place. This connection $C D_{G}$ will create a continuous connection across the network, depending on its coverage range $C_{R}$.

$$
C D_{G}=\left\{C_{R}\right\}
$$

Each node has different $C_{R}$; therefore, the formed DG will be of different sizes. Thus, after dividing the data sets into groups, the neighbor sets for each sensor are divided based on these feature divisions. Also, the sensor in that neighboring set will detect the sensor that has the most energy $E$. Then, find the distances $D_{\text {ist }}$ between the sensors and order the neighbor sets according to that distance $D_{i s t}$ and $E$ within its coverage area $C_{R}$.

$$
E=I_{E}-E_{E}
$$

Also, find the number of sensors $N_{S}$ in that neighbor set. The $D_{i s t}$ is calculated according to the Pythagorean Theorem, here, location of the sensor $\left(X_{1}, Y_{1}\right)$, and its neighbor location is $\left(X_{2}, Y_{2}\right)$, the distance $D_{i s t}$ between the two sensors are then computed as below,

$$
D_{i s t}=\sqrt{\left|X_{1}-X_{2}^{2}+\right| Y_{1}-Y_{2}^{2}}
$$

We formed the most perfect $N_{S}$ with the smallest and maximum size of its features to calculate these distances. Each sensor is maintained $N_{L}$ in that the distance information is stored, and it will be updated frequently according to the dynamic changes.

$$
N_{S}=\mid\left(s_{i} \mid D_{i s t} \leq C_{R}\right)
$$

The number of nodes in each sensor must be constantly monitored for changes in the network. Only by updating the setbacks that occur can group according to the data, according to these changes, the neighborhood connectivity of the network is transformed. The changes in it can be calculated Lagrange method, and small changes in the network and significant changes can be predicted. If a group is set up with specific data, it cannot contain data with other features. The sensors also calculate the value of energy expended $E_{E}$ during each contact and set the durable group accordingly to enhance the network life, reducing network losses. Here $T_{X P}$ is the transmission packet's energy and $R_{X P}$ is the receiving packet energy, respectively. $I_{E}$ Described the initial energy level of the sensor.

$$
E_{E}=I_{E}-\left(T_{X P}+R_{X P}\right)
$$


With these calculations, a highly accurate $D_{G}$ and network connectivity was established. In addition, the neural network system is used to detect changes in network sensing. It forms a training set process $T_{P}$ in the network, receives all the fuzzified data, sends it from the input layers to the hidden layer, and starts processing. In this neural network, before the data is given in the input layers, each data is provided a weight $W$ value and tuned; when processed in a hidden layer, the threshold $T_{H}$ sizes are marked.

$$
T_{P}=\sum \Upsilon_{i j} a\left(I_{P} I_{W}+T_{H}\right) \rightarrow \text { output }
$$

Here $a()$ is the neural stimulation process $I_{W}, I_{P}$ the input layer data, and the input weight assignment process in order. The hidden layer processing limitations are known from the hidden layer's threshold $T_{H}$ and $\Upsilon_{i j}$ the link weight between the hidden layers to output $H_{O P Y_{i j}}$. If the error differences are small, the network area can be predicted to be consistent for a given period as the training samples of the $S_{D}$ are constantly monitored. In that time, the hidden layer processed outputs $H_{O P}$ declared as

$$
H_{O P}=H_{O P Y_{i j}}
$$

The number of neurons in the hidden layers is less than the number of neurons in the training set. The highlight of machine learning is learning the transactions that occur on the network in a brief period and running it back and forth in a straightforward way so that each node realizes and acts on the network interactions. The neural calculations calculated here are machine learning methods. The given inputs and the weight $W$ values used to tune it are converted to machine learning mode in this mode. Due to this, its environmental prediction accurateness is very high. This way, we do not have to iterate the inputs multiple times.

In addition, the BAT computational system is used to regulate data transmission. Path optimization is implemented based on the bird's behavior, such as pulse, emission, echo, and loud noise.

\subsection{Optimized Route Selection}

In this calculation, the sensor's location $L_{i}$ runs with the BAT computation is identified, and its moving velocity is $S_{i}$ and time $T$ also verified. In addition, update this sensor $L$ and velocity variations at each time as follows.

$$
\begin{aligned}
L_{i} & =N_{B}\left(L_{i-1}-L\right) \\
N_{B} & =N_{B M I}+\left(N_{B M A}-N_{B M I}\right) \Upsilon
\end{aligned}
$$

Here, $N_{B}$ indicated the node buffer minimum $N_{B M I}$ and maximum $N_{B M A}$ level provided the awareness of the network environment state. Later update the velocity $S_{i}$ of the sensor is:

$$
S_{i}=S_{i}+\left(L-L_{I-1}\right) \Upsilon
$$

Subsequently, BAT computation monitors changes in the sensor; accordingly, its inactive moving $I_{M}$ state is calculated. Here, $M_{p M A}$ Is the sensor's maximum $M_{p M I}$ and minimum $I_{M}$ state and $R$ the number of repetitions in the computations accurate $I_{M}$.

$$
I_{M}=\frac{R\left(M_{p M A}-M_{p M I}\right)}{R}
$$

According to the moving velocity of the sensor, the global solution $G_{S}$ determined as follows:

$$
G_{S}=L_{i}+S_{i}+I_{M}
$$

Later, after the determination of $G_{S}$ sensor location, the new location $L_{i N}$ of the sensor updated as follows:

$$
L_{i N}=L_{i n}+\alpha P_{S}
$$

Here $L_{i n}$ defined the sensor's new location and $P_{S}$ explained the sensor's population during the network changes with the tuning parameter $\alpha$ at a time described as $0-1$. During this time, the data transmission rate $D_{T R}$ and noise 
values are updated at each repetition process. Consequently, in the locality of the destination, the noise will slowly reduce because of $D_{T R}$ will increase gradually as computed as below. Here is the packet size $S_{P}$ of data packets.

$$
D_{T R}=S_{P} D_{T R}
$$

This proposed path selection system is set up with the events of the network in which the local and global forwarder selection for the route selection takes place through the BAT calculation itself. Thus, the given parameters pave the way with the most consistent local and global searches in a very short time.

\subsection{Routing Method of Proposed Model}

All the nodes are organized at the beginning of the network, and network communication is established to transfer the packets. In this proposed method, initially, we used $F_{I}$ and neuron systems to categorize the $D_{G}$ as a cluster. In this way, more $D_{G}$ were created to identify the specific features of the data. To classify this, the network used few parameters such as node location, sensing counts, sensing time, and ID to filter the features. All these parameters are processed $F_{I}$, and the fuzzy set's output is transferred to the neural network to get more accurate $D_{G}$. Also, the node with the shortest distance and high energy towards the destination is chosen as neighbor sets within the coverage range of each sensor. The $D_{G}$ will be changed as per the environmental state and node location variation, so the network will be updated to reconstruct the data group according to the changes. Due to the continuous monitoring sensor predicted the status of the incoming data. It changed the $D_{G}$. Accordingly, the nodes can perform computations to notice the environment continues in the proposed method. The BAT computation predicts those forwarder nodes' local and global quality to build the routing path.

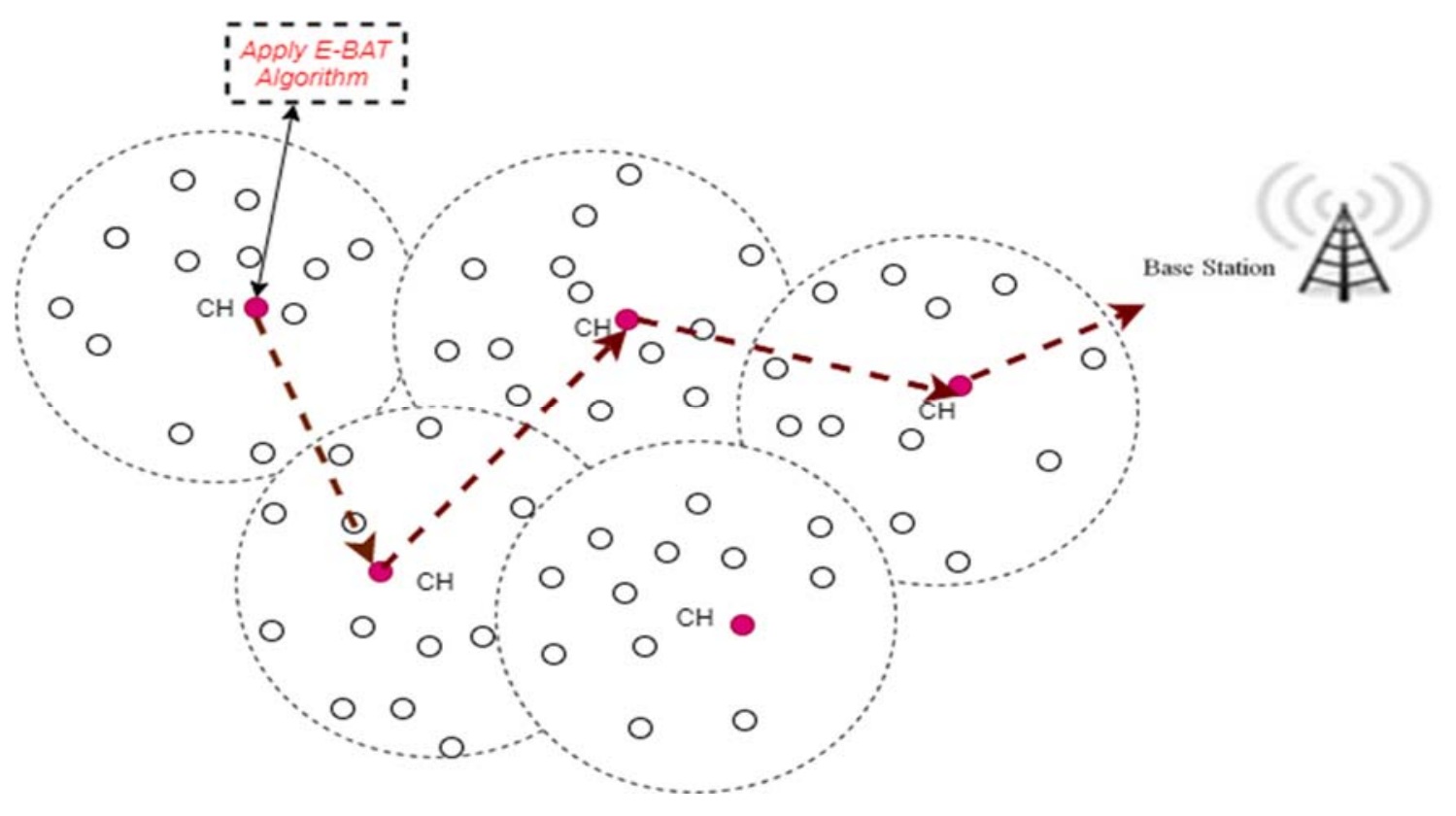

Fig.2. Forwarder Selection

The Enhanced BAT computation is used to determine the optimal Forwarder routing path, as shown in the diagram above. The solutions given by the EBAT Computation are evaluated using an unique Fitness Function. The fittest solution offers details on the most efficient route to the Base Station.

\section{Results and Discussion}

Nodes in this network have been changed from 100 to 200 and tested. The network area is chosen to be between 200 and 200, and the nodes are randomly placed in it. Random data nodes sense application as it travels. Its performances are given below in graph form. In this, the network parameters used are shown in Table.1 below. 


\begin{tabular}{|l|l||}
\hline Parameter & Value \\
\hline \hline Traffic Type & Sense Application \\
\hline \hline Nodes & $100-200$ \\
\hline \hline Propagation Model & TwoRayGround \\
\hline \hline Antenna & Omni-Directional \\
\hline \hline Network Size & $200 \times 200$ \\
\hline
\end{tabular}

Table.1. Network Parameters

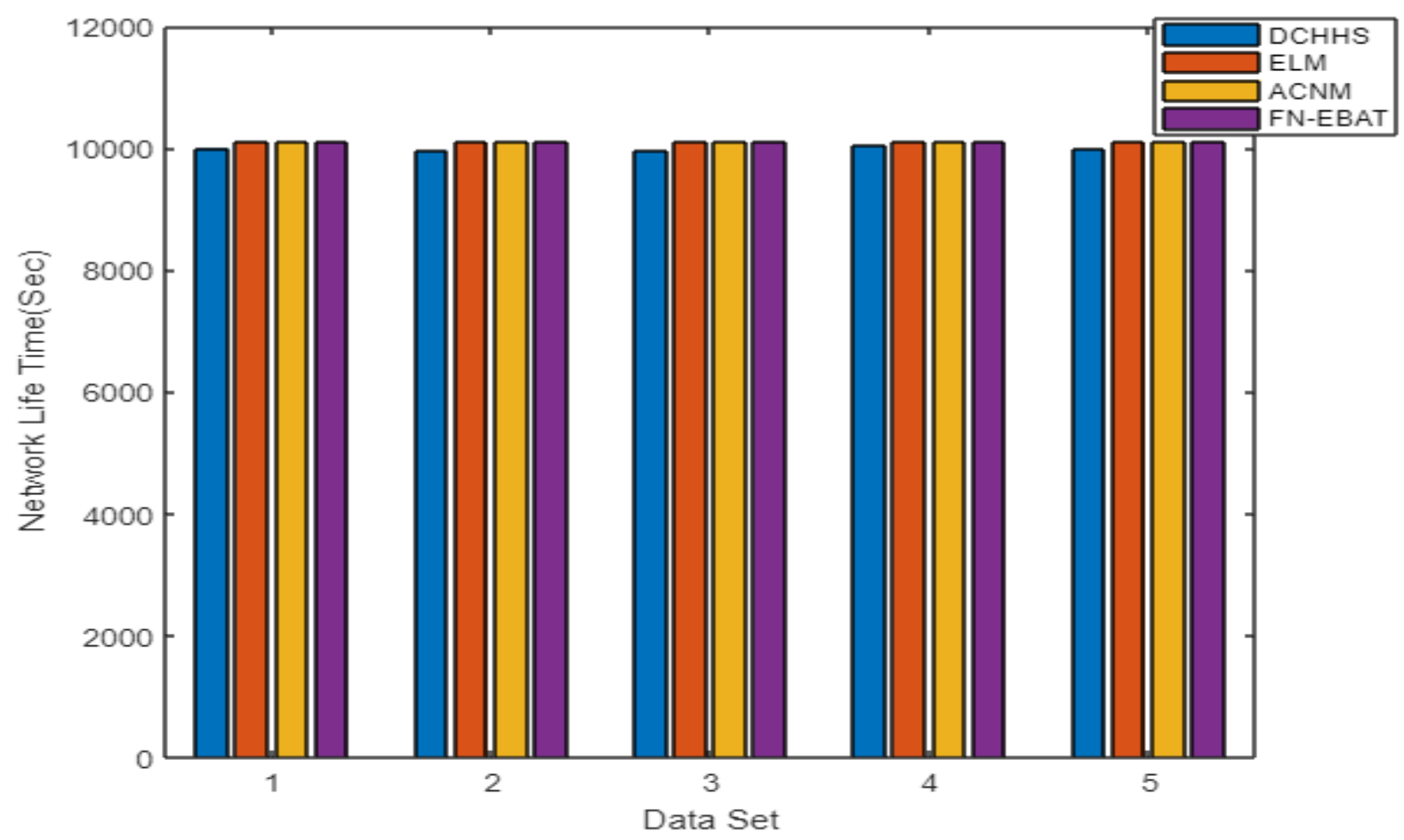

Fig.3. Dataset Vs. Network Lifetime

Network lifetime is the amount of life that nodes in the network use and extend depending on energy. If the network has nodes with a longer lifespan, the performance of that network will be longer. If the energy is used for a long time, the routing on the network can be considered better. The proposed model, FN-EBAT, has a longer lifespan than other ACNM, ELM, and DCHHS protocols, as shown in Figure.3. As it is a sensor network, the neighbor set is formed by special information of the data perceived in the network. The data is then selected for optimized routing. As a result, the energy losses are minimized, and the data transmission is minimized, and the lifespan is extended. 


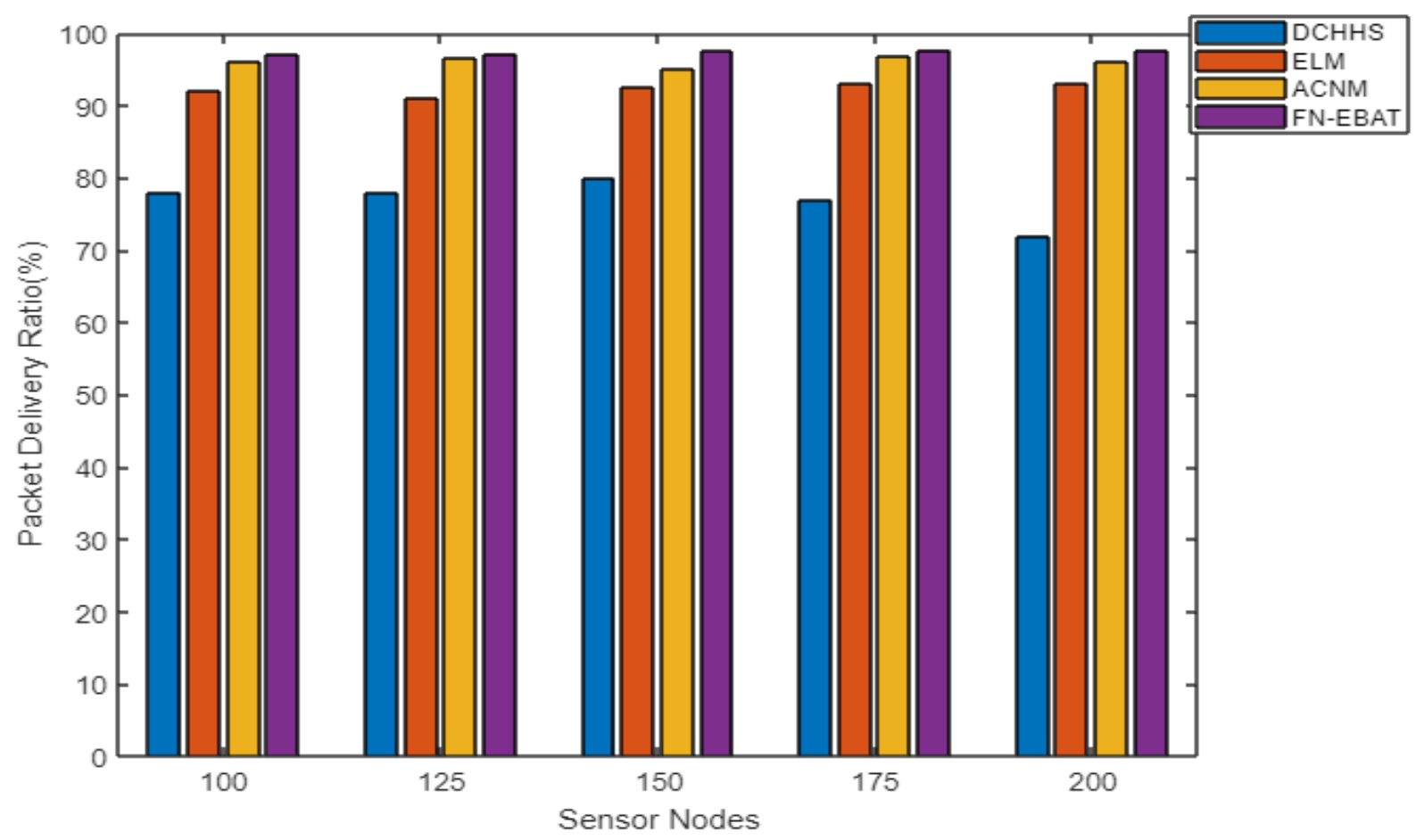

Fig.4. No.of Sensor Nodes Vs. Packet Delivery Ratio

The packet delivery ratio -PDR is the percentage of data packets delivered to the destination. This will increase if the network communication is working well. Otherwise, the percentage will start to decrease. The main reason for this is both routing and neighbor selection. Here, in FN-EBAT, PDR is higher than other protocols, as shown in Figure.4. This is because the data groups are made from the neighbor set, and then the accurate routing is selected. Many network factors such as energy, data types, distance, etc., are chosen, and the features for that data are considered, and then the neighbors are selected. Also, routing is created through BAT optimization. As a result, the percentage of data delivery has increased as a stable network has been created.

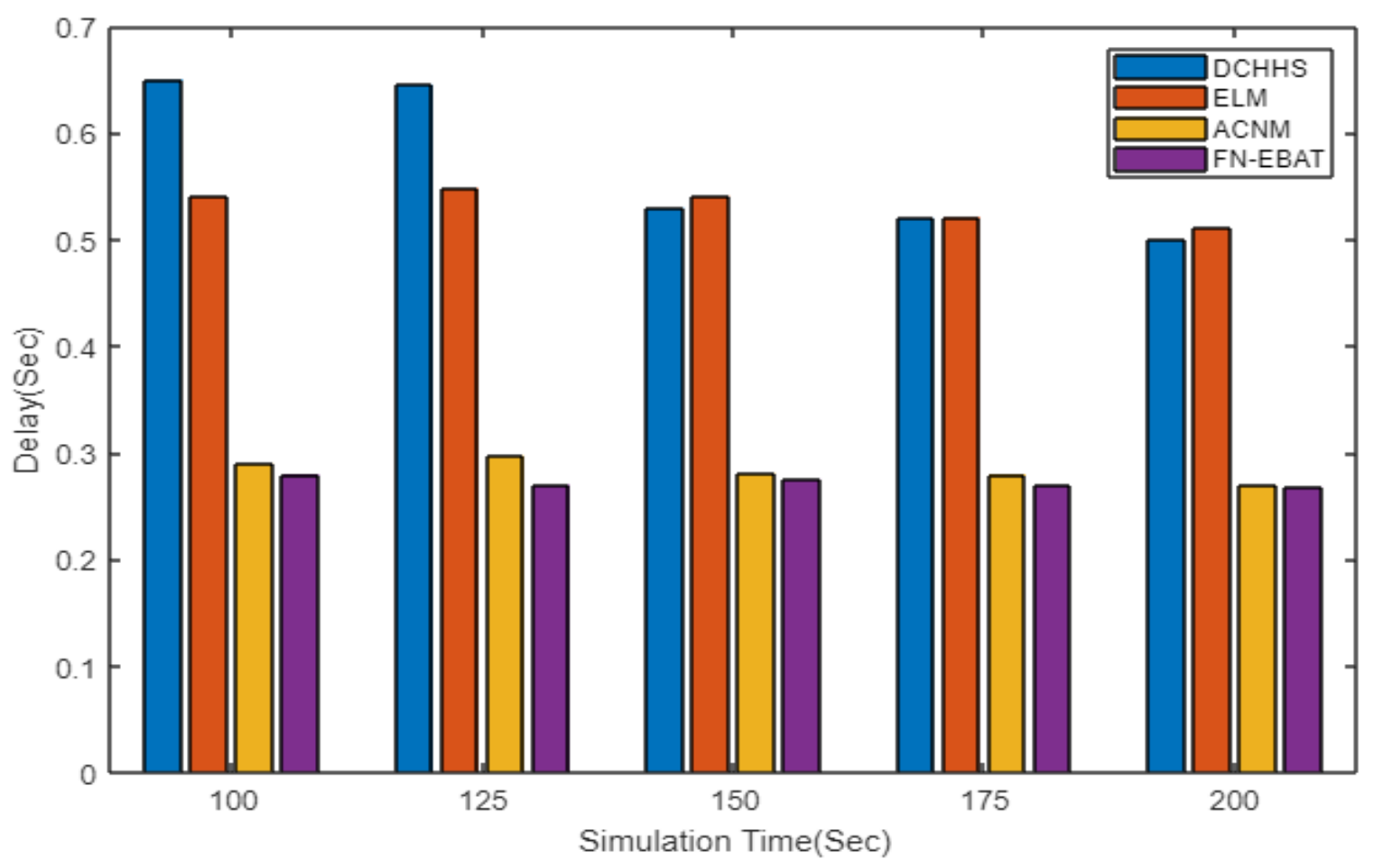

Fig.5. Simulation Time Vs. Delay 
Network Delay is the time delay that occurs during transmission. This will vary depending on the stability of the network. Many factors, such as the nature of the wireless channel, the connectivity period of the nodes, and the packet buffering period, determine this network delay. If the delay is short, the protocol running on the network can be considered running accurately. In this FN-EBAT method, the selection of neighbors is accurate as the data is divided according to its feature sets and then routed. Thus it can be seen that the performance of the proposed has increased Figure.5.

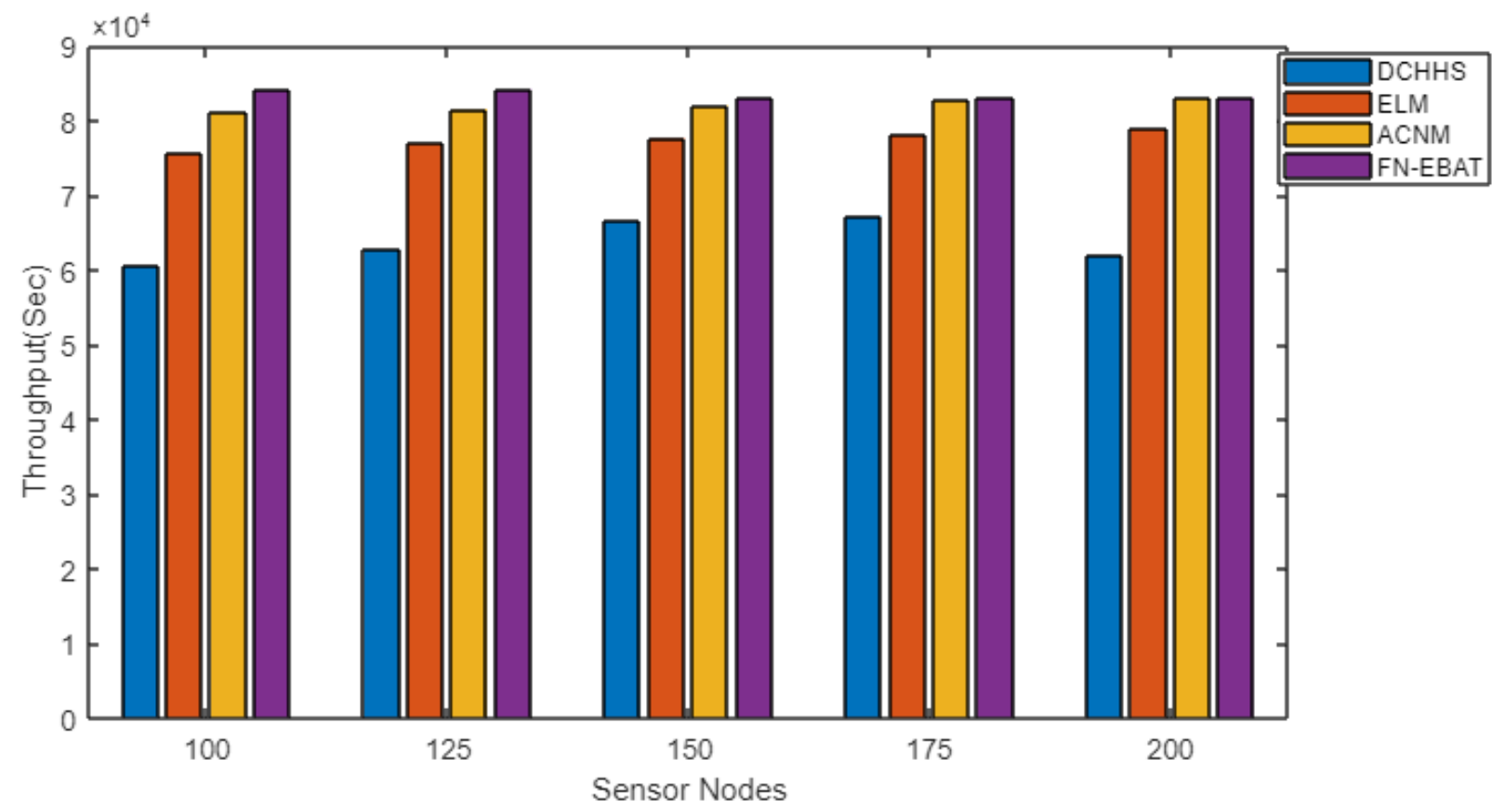

Fig.6. No.of Sensor Nodes Vs. Throughput

Throughput is the number of packet bits that receive the destination. If the functions of the network are consistent, the packets will quickly go to the destination. Then, the number of its bits will also increase. Throughput is one of the qualities of measurements of a network. Here we can see that the throughput of the proposed method FNEBAT is high, as shown in Figure.6. These variations can be seen due to optimized group formation and routing selection. This includes calculating fuzzy, neural, and BAT computations, making the wireless communication sensor network better.

\section{Conclusion and Future work}

When the sensor senses the data somewhere in the network and sends it to the destination elsewhere, the network encounters various problems. To meet these requirements, the sensors in the networks require a proper protocol. The FN-EBAT data group designed here selects the path and then. The sensors use the fuzzy-neuron method to form a data group and the BAT computations to choose the routing path. The sensors use several parameters to separate the data features and create accurate data groups. This will not confuse aggregating the data.Similarly, the forwarder selection required to forward the data is made by BAT computation. This reduces the cost of energy by selecting local and global best forwarders and transmitting data through them. In the future, secure routing of data transmission on this network will enable secure data transmission.

\section{References}

[1] Neeraj Kumar, Manoj Kumar, R.B. Patel, "Coverage and Connectivity Aware Neural Network Based Energy Efficient Routing in Wireless Sensor Networks",Journal on Applications of Graph Theory in Wireless Ad hoc Networks and sensor Network(JGRAPHADHOC), Vol 2,No 1,March 2010.

[2] Mayur V. Bhanderi and Hitesh B. Shah, "Machine Learning for Wireless Sensor Network: A Review, Challenges and Applications",Advance in Electronic and Electric Engineering,Volume 4, Number 52014.

[3] Manomi K S, Vinutha C B, M Z Kurian, "Design Approach for Increased Lifetime of WSN using Artificial Neural Network Based Data Aggregation",International Journal of Advanced Networking \& Applications (IJANA).

[4] Raja Vara Prasad Y, Rajalakshmi Pachamuthu, "Neural Network based Short Term Forecasting Engine To Optimize Energy And Big Data Storage Resources Of Wireless Sensor Networks",IEEE 39th Annual International Computers, Software \& Applications Conference, 2015 .

[5] Abdulaziz Y. Barnawi and Ismail M. Keshta, "Energy Management in Wireless Sensor Networks Based on Naive Bayes, MLP, and SVM Classifications: A Comparative Study",Hindawi Publishing Corporation Journal of Sensors Volume 2016. 
[6] Mr.Maharajan M S, T.Abirami, "An Energy Efficient Mechanism using Mutated Bat Algorithm in Wireless Sensor Network",International Journal of Innovative Technology and Exploring Engineering (IJITEE),Volume-8 Issue-11, September 2019.

[7] Kavita, Dr. Ramesh Chand Kashyap, "Improved Bat Algorithm Based Clustering in WSN",International Journal of Engineering Development and Research( IJEDR), Volume 4, Issue 4,2016.

[8] Asad Awan, Suresh Jagannathan, Ananth Grama, "Macroprogramming Heterogeneous Sensor Networks Using COSMOS".

[9] Georgios Smaragdakis, Ibrahim Matta, Azer Bestavros, "SEP: A Stable Election Protocol for clustered heterogeneous wireless sensor networks".

[10] Mohammed Al-Medhwahi, Fazirulhisyam Hashim, Borhanuddin Mohd Ali, A Sali and Abdulsalam Alkholidi, "Resource allocation in heterogeneous cognitive radio sensor networks",International Journal of Distributed Sensor Networks,Vol. 15(7),2019.

[11] Trupti. M. Behera, Sushanta. K. Mohapatra, Umesh. C. Samal, Mohammad. S. Khan, "Hybrid Heterogeneous Routing Scheme for Improved Network Performance in WSNs for Animal Tracking".

[12] [12] Joel B. Predd, Sanjeev R. Kulkarni, and H. Vincent Poor, "Distributed Learning in Wireless Sensor Networks",IEEE Signal Processing Magazine July 2006.

[13] Michael Matusowsky, Daniel T. Ramotsoela and Adnan M. Abu-Mahfouz, "Data Imputation in Wireless Sensor Networks Using a Machine Learning-Based Virtual Sensor",Journal of Sensor and Actuator Networks 27 May 2020.

[14] Trong-The Nguyen, Jeng-Shyang Pan and Thi-Kien Dao, "A Compact Bat Algorithm for Unequal Clustering in Wireless Sensor Networks",14 May 2019.

[15] Ki-Seong Lee, Sun-Ro Lee, Youngmin Kim and Chan-Gun Lee, "Deep learning-based real-time query processing for wireless sensor network",International Journal of Distributed Sensor Networks, Vol. 13(5), 2017.

[16] Nimmagadda Srilakshmi and Arun Kumar Sangaiah, "Selection of Machine Learning Techniques for Network Lifetime Parameters and Synchronization Issues in Wireless Networks",Journal of Information Processing Systems, April 18, 2019.

[17] MR. Sachin, B. Jadhav, "Machine Learning Aspects in Wireless Sensor Network",Iconic Research and Engineering Journals, Volume 2 Issue 1,MAY 2019.

\section{Authors Profile}

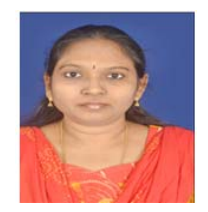

C.Sudha is currently a Part Time Research Scholar in CSE Department at Annamalai University, Chidambaram, and Tamilnadu. She completed her M. E in CSE from Anna University Regional center, Coimbatore in 2013. She Received. her B.E degree in CSE from Sona college of Technology, Salem. Currently she is working as an Assistant Professor at Mahatma Gandhi Institute of Technology, Hyderabad. Her Research interest includes Computer Networks, Wireless Sensor Networks, IoT based Architectures.

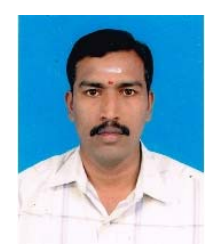

Dr D. Suresh received the B.E (IT)., degree in Mohamed Sathak Engineering College in 2004. He received M.E degree in Computer Science and Engineering from the Annamalai University in 2008. He has been with Annamalai University, since 2005. He completed his Ph.D degree in Computer Science and Engineering at Annamalai University, in the year 2015. He is currently an Assistant Professor in Information Technology at Annamalai University. He published 20 papers in international conferences and journals. His research interest includes Mobile Networks, Network Security, Wireless Sensor Networks and Network Simulator.

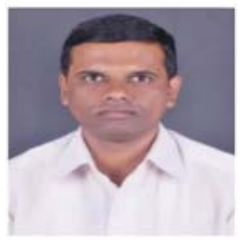

Dr A.Nagesh is currently working as a professor in CSE at MGIT, Hyderabad. He completed B.E and M. Tech from Osmania University, Hyderabad in 1996 and 2002 respectively. He completed his Ph. D in CSE from JNTUH, Hyderabad in the year 2012. He is having total 22 years of teaching experience. Presently he is supervising five Ph. D students. He published 40 papers in national \& international journals. His research areas include pattern recognition, speech processing and data mining. 